\title{
INTEGER-VALUED POLYNOMIALS ON A SUBSET
}

\author{
PAUL-JEAN CAHEN
}

(Communicated by Louis J. Ratliff, Jr.)

\begin{abstract}
We let $D$ be a local (noetherian) one-dimensional unibranched domain, $K$ its quotient field, $m$ its maximal ideal, $D^{\prime}$ its integral closure, and $\mathfrak{m}^{\prime}$ the maximal ideal of $D^{\prime}$. If $E$ is a subset of $K$, we let $\operatorname{Int}(E, D)$ be the set of integer-valued polynomials on $E$, thus $\operatorname{Int}(E, D)=\{f \in K[X] \mid f(E) \subset D\}$. For a fractional subset $E$ of $D$ (i.e., there is a nonzero element $d$ of $D$ such that $d E \subset D)$, we show that the prime ideals of $\operatorname{Int}(E, D)$ above $m$ are in one-to-one correspondence with the elements of the topological closure of $E$ in the completion of $K$ for the $\mathrm{m}^{\prime}$-adic topology.
\end{abstract}

\section{INTRODUCTION}

Throughout this paper, $D$ is a domain; we denote by $K$ its quotient field and by $D^{\prime}$ the integral closure of $D$. If $E$ is a subset of $K$, we let $\operatorname{Int}(E, D)$ be the set of integer-valued polynomials on $E$, thus $\operatorname{Int}(E, D)=\{f \in K[X] \mid f(E) \subset$ $D\}$; it is clearly a ring containing $D$, throughout, we assume that $E$ is nonempty and $D$ is not a field, hence that $\operatorname{Int}(E, D)$ is strictly contained in $K[X]$ (as indeed $\operatorname{Int}(E, D) \cap K=D)$. If $E$ is $D$ itself, we simply write $\operatorname{Int}(D)$ for $\operatorname{Int}(D, D)$.

The very classical case is that of The Ring of integer-valued polynomials, i.e., $\operatorname{Int}(\mathbb{Z})$, or even $\operatorname{Int}(D)$ where $D$ is the ring of integers of a number field $[22,23]$ and, even more generally, where $D$ is Dedekind [5]; by localization, $D$ turns into a discrete rank-one valuation domain and the prime ideals of Int $(D)$ above the maximal ideal $\mathfrak{m}$ of $D$ are then known to be in one-to-one correspondence with the elements of the completion $\widehat{D}$ of $D$ : to any element $\alpha$ of $\widehat{D}$ corresponds the prime $\mathfrak{M}_{\alpha}=\{f \in \operatorname{Int}(D) \mid f(\alpha) \in \widehat{\mathfrak{m}}\}[12,4,13]$, considering the $\mathrm{m}$-adic topology, this result generalizes to the case where $D$ is noetherian, local, one-dimensional, with finite residue field, and is analytically irreducible (that is where $\widehat{D}$ is a domain) [11]. Dropping this last hypothesis, however, only two facts were known in the previous decade:

Received by the editors March 26, 1991 and, in revised form, July 17, 1991; presented at Integer-valued Polynomials Encounter, December 12, 1990, Centre International de Rencontres Mathématiques, Marseille.

1991 Mathematics Subject Classification. Primary 13B25; Secondary 11S05, 12J10, 13E05, $13 \mathrm{G} 05$.

The author wishes to thank the referee for several useful comments and suggestions. 
(1) The prime of $\operatorname{Int}(D)$ above $\mathfrak{m}$ are of the type $\mathfrak{M}_{\alpha}=\{f \in \operatorname{Int}(D) \mid f(\alpha) \in$ $\widehat{\mathfrak{m}}\}$ [13].

(2) If $D$ is not analytically irreducible, then some of those primes are equal; namely, if $(\alpha-\beta)$ is a zero divisor in $\widehat{D}$, then $\mathfrak{M}_{\alpha}=\mathfrak{M}_{\beta}[14]$.

Letting $D$ be noetherian, one-dimensional, and local, we say that $D$ is unibranched if, moreover, $D^{\prime}$ is local (hence a discrete rank-one valuation domain); recall that $D$ is analytically irreducible if and only if it is unibranched and $D^{\prime}$ is a finite $D$-module $[3,21]$. Very recently it has been shown that if $D$ is not unibranched (first in the case where $D^{\prime}$ is a finite $D$-module [9] and then without this hypothesis [19]), there are only finitely many primes above $\mathfrak{m}$ (namely, $\mathfrak{M}_{\alpha}=\mathfrak{M}_{\beta}$ if $(\alpha-\beta)$ lies in some nontrivial ideal of $D$ ); hence the unibranched (and nonanalytically irreducible) case was the only one to remain open. So, letting $D$ be a unibranched domain (with finite residue field), we determine here entirely the spectrum of $\operatorname{Int}(E, D)$ for any fractional subset $E$ of $D$ (i.e., such that $d E \subset D$ for a nonzero element $d$ of $D$ ), recovering the spectrum of $\operatorname{Int}(D)$ (i.e., $E=D$ ) as well as the classical results on a discrete rank-one valuation domain (i.e., $D=D^{\prime}$ ) as special cases.

In the first section we state some generalities on integer-valued polynomials on a subset, mostly on Krull dimension and localization. In $\S 2$, generalizing a result of Gilmer, Heinzer, and Lantz [19], we show that if $D$ is noetherian, onedimensional, local, with finite residue field, then, for any fractional subset $E$ of $D, \operatorname{Int}\left(E, D^{\prime}\right)$ is the integral closure of $\operatorname{Int}(E, D)$ and that if, moreover, $D$ is unibranched then $\operatorname{Int}\left(E, D^{\prime}\right)$ is a radical extension of $\operatorname{Int}(E, D)$ (i.e., every element of $\operatorname{Int}\left(E, D^{\prime}\right)$ has a power in $\left.\operatorname{Int}(E, D)\right)$. In $\S 3$ we first determine the spectrum of $\operatorname{Int}(E, D)$ for a fractional subset $E$ of a discrete rank-one valuation domain and then of a unibranched domain $D$ (whose integral closure $D^{\prime}$ is a discrete rank-one valuation domain) as follows: Letting $\mathfrak{m}^{\prime}$ be the maximal ideal of $D^{\prime}$, we consider the $\mathfrak{m}^{\prime}$-adic topology, hence the topology defined by the valuation of $D^{\prime}$, rather than the m-adic topology, treating $D$ as a subset of $D^{\prime}$; we can thus deduce the spectrum of $\operatorname{Int}(D)$ from that of $\operatorname{Int}\left(D, D^{\prime}\right)$ and more generally establish that for any fractional subset $E$ of $D$ the primes of $\operatorname{Int}(E, D)$ above $\mathfrak{m}$ are in one-to-one correspondence with the elements of the topological closure of $E$ in the completion of $K$ for the topology defined by the valuation of $D^{\prime}$. Lastly we discuss the noetherian property of $\operatorname{Int}(E, D)$, generalizing and shedding some light on the results of [19] obtained in the particular case of $\operatorname{Int}(D)$.

\section{KRULl Dimension AND localization}

We start with some elementary inclusions.

Proposition 1.1. If $D$ and $B$ are two domains of quotient field $K$ such that $D \subset B$ and $E, F$ two subsets of $K$ and such that $E \subset F$, then $\operatorname{Int}(F, D) \subset$ $\operatorname{Int}(E, B)$.

If $E$ is not contained in $D$ then the polynomial $X$ is not in $\operatorname{Int}(E, D)$, thus:

Proposition 1.2. Let $D$ be a domain and $E$ a subset of $K$. Then the following statements are equivalent:

(i) $E \subset D$. 
(ii) $\operatorname{Int}(D) \subset \operatorname{Int}(E, D)$.

(iii) $D[X] \subset \operatorname{Int}(E, D)$.

It is easy to describe some primes of $\operatorname{Int}(E, D):$ If $\alpha \in E$ and $\mathfrak{p}$ is a prime ideal of $D$, we note $\mathfrak{P}(\alpha, \mathfrak{p})=\{f \in \operatorname{Int}(E, D) \mid f(\alpha) \in \mathfrak{p}\}$ the set of polynomials of $\operatorname{Int}(E, D)$ taking on the value of $\alpha$ in $\mathfrak{p}$; this set is clearly a prime ideal of $\operatorname{Int}(E, D)$ above $\mathfrak{p}$ and the quotient $\operatorname{Int}(E, D) / \mathfrak{P}(\alpha, \mathfrak{p})$ is isomorphic to $D / \mathfrak{p}$; in particular, if $\mathfrak{m}$ is a maximal ideal of $D$ then $\mathfrak{M}_{\alpha}=\mathfrak{P}(\alpha, \mathfrak{m})$ is a maximal ideal of $\operatorname{Int}(E, D)$. If $\mathfrak{p} \subsetneq \mathfrak{q}$ then $\mathfrak{P}(\alpha, \mathfrak{p}) \subsetneq \mathfrak{P}(\alpha, \mathfrak{q})$, and we can generalize here to $\operatorname{Int}(E, D)$ the results known for the Krull dimension of $\operatorname{Int}(D)[5,13]$ (we denote by $\operatorname{dim} R$ the Krull dimension of a ring $R$ ).

First note that if $E$ is too large it may happen that every polynomial in $\operatorname{Int}(E, D)$ is constant (i.e., $\operatorname{Int}(E, D)=D)$. Also, following McQuillan [20], we shall say that $E$ is a fractional subset of $D$ if there exists a nonzero element $d$ of $D$ such that $d E \subset D$.

Proposition 1.3. Let $D$ be a domain and $E$ a subset of $K$. Then $\operatorname{dim} \operatorname{Int}(E, D)$ $\geq \operatorname{dim} D ;$ if, moreover, $E$ is a fractional subset of $D$ then $\operatorname{dim} \operatorname{Int}(E, D) \geq$ $\operatorname{dim} D+1$.

Proof. Let $(0)=\mathfrak{p}_{0} \subsetneq \mathfrak{p}_{1} \subsetneq \cdots \subsetneq \mathfrak{p}_{d}$ be a chain of primes of $D$; it gives rise to the chain of primes $\mathfrak{P}\left(\alpha, \mathfrak{p}_{0}\right) \subsetneq \mathfrak{P}\left(\alpha, \mathfrak{p}_{1}\right) \subsetneq \cdots \subsetneq \mathfrak{P}\left(\alpha, \mathfrak{p}_{d}\right)$ of $\operatorname{Int}(E, D)$. Moreover, if $d$ is such that $d E \subset D$ then $d(X-\alpha) \in \mathfrak{P}\left(\alpha, \mathfrak{p}_{0}\right)$, hence $(0) \subsetneq$ $\mathfrak{P}\left(\alpha, \mathfrak{p}_{0}\right)$.

If $R$ is a domain, recall that its valuative dimension $\operatorname{dim}_{\mathrm{v}} R$ is the supremum of the Krull dimensions of the overrings of $R$ in the quotient field of $R$; Also recall that a Jaffard domain is a domain such that $\operatorname{dim}_{\mathrm{v}} R=\operatorname{dim} R$; and lastly recall that a noetherian domain is a Jaffard domain [1].

Corollary 1.4. Let $D$ be a domain and $E$ a subset of $K$. Then

(i) $\operatorname{dim} \operatorname{Int}(E, D) \leq \operatorname{dim}_{\mathrm{v}} D+1$;

(ii) if $D$ is a Jaffard domain then $\operatorname{dim} \operatorname{Int}(E, D) \leq \operatorname{dim} D+1$;

(iii) if $D$ is a Jaffard domain and $E$ a fractional subset of $D$, then $\operatorname{dim} \operatorname{Int}(E, D)=\operatorname{dim} D+1$.

Proof. (i) $\operatorname{Int}(E, D)$ satisfies the inclusions $D \subset \operatorname{Int}(E, D) \subset K[X]$, and the result follows from [2, Lemma 1.1]; (ii) and (iii) are clear.

We conclude this section with localization properties:

Proposition 1.5. Let $D$ be a domain, $E$ a subset of $K$, and $S$ a multiplicative subset of $D$. Then

(i) $S^{-1} \operatorname{Int}(E, D) \subset \operatorname{Int}\left(E, S^{-1} D\right)$;

(ii) if $E$ is a subset of $D$ and $D$ is noetherian, then $S^{-1} \operatorname{Int}(E, D)=$ $\operatorname{Int}\left(E, S^{-1} D\right)$;

(iii) if $A=E$ is a subring of $D$ and $S$ is a multiplicative subset of $A$, then $S^{-1} \operatorname{Int}(A, D) \subset \operatorname{Int}\left(S^{-1} A, S^{-1} D\right)$. Moreover, if $A$ or $D$ is noetherian, then $S^{-1} \operatorname{Int}(A, D)=\operatorname{Int}\left(S^{-1} A, S^{-1} D\right)=\operatorname{Int}\left(A, S^{-1} D\right)$.

Proof. (i) Immediate. 
(ii) Let $f \in \operatorname{Int}\left(E, S^{-1} D\right)$. The $D$-module generated by $f(E)$ is (included in) a finite $D$-module (generated by the coefficients of $f$ ); hence there exists $s \in S$ such that $s f(E) \subset S^{-1} D$, thus $f \in S^{-1} \operatorname{Int}(E, D)$.

(iii) If $f \in \operatorname{Int}(A, D), f$ is by definition a polynomial with coefficients in $K[X]$ whose homomorphic image in $(K / D)[X]$ is null on $A$, then its image in $S^{-1}(K / D)[X]$ is null on $S^{-1} A$ [10, Proposition 4], thus $\operatorname{Int}(A, D) \subset$ $\operatorname{Int}\left(S^{-1} A, S^{-1} D\right)$ and the following inclusions always hold: $S^{-1} \operatorname{Int}(A, D) \subset$ $\operatorname{Int}\left(S^{-1} A, S^{-1} D\right) \subset \operatorname{Int}\left(A, S^{-1} D\right)$. Moreover, if $D$ is noetherian it follows from (ii) that $\operatorname{Int}\left(A, S^{-1} D\right) \subset S^{-1} \operatorname{Int}(A, D)$; the same holds if $A$ is noetherian, with the same proof, considering $A$-modules instead of $D$-modules.

Remark 1.6. If $E$ is simply a subset of $K$ (and not of $D$ ), then $f(E)$ is not necessarily included in the $D$-module generated by the coefficients of $f$ and the conclusion of (ii) may fail. Indeed if, for instance, $D=\mathbb{Z}, S$ is any nontrivial multiplicative set, and $E=S^{-1} \mathbb{Z}$, then $\operatorname{Int}(E, \mathbb{Z})=\mathbb{Z}[20$, Lemma 2.0], whereas $\operatorname{Int}\left(E, S^{-1} \mathbb{Z}\right)=\operatorname{Int}\left(S^{-1} \mathbb{Z}\right)$ does contain nonconstant polynomials.

\section{INTEGRAL CLOSURE}

We start with a general condition for $\operatorname{Int}(E, D)$ to be integrally closed.

Proposition 2.1. Let $D$ be a domain and $E$ a subset of $K$. Then $\operatorname{Int}(E, D)$ is integrally closed if and only if $D$ is integrally closed.

Proof. - If $D$ is not integrally closed there exists $x \in K, x \notin D$, which is integral over $D$, then $x \notin \operatorname{Int}(E, D)$ but $x$ is integral over $\operatorname{Int}(E, D)$.

- If $D$ is integrally closed, and if $f \in K(X)$ is integral over $\operatorname{Int}(E, D)$, then $f \in K[X]$ (since $f$ is integral over $K[X]$, which is integrally closed), and, for every $x \in E, f(x)$ is integral over $D$; hence $f(x) \in D$, thus $f \in$ $\operatorname{Int}(E, D)$.

In [19] it is proved that if $D$ is a one-dimensional noetherian domain then the elements of $\operatorname{Int}\left(D^{\prime}\right)$ are integral over $\operatorname{Int}(D)$; this is done by assuming (without loss of generality) that $D$ is local. Here we state a similar result for $\operatorname{Int}(E, D)$, but first we present the following

Lemma 2.2. Let $D$ be a noetherian domain, $E$ a fractional subset of $D$, and $f \in \operatorname{Int}\left(E, D^{\prime}\right)$. Then $f(E)$ is contained in a $D$-algebra $R$, finitely generated as a $D$-module and such that $D \subset R \subset D^{\prime}$.

Proof. Let $d$ be a nonzero element such that $d E \subset D$, and let $f=a_{0} / b+$ $\left(a_{1} / b\right) X+\cdots+\left(a_{n} / b\right) X^{n}$, where $a_{0}, a_{1}, \ldots, a_{n}$ and $b$ are in $D$. If $x \in$ $E$ it is clear that $b d^{n} f(x) \in D$, hence $f(E)$ is included in the $D$-module $M=D^{\prime} \cap\left(1 / b d^{n}\right) D$. Since $D$ is noetherian, $M$ has finitely many generators $x_{1}, \ldots, x_{r}$ and $f(E)$ is included in the $D$-algebra $R=D\left[x_{1}, \ldots, x_{r}\right]$, which is also a finitely generated $D$-module.

Proposition 2.3. Let $D$ be a one-dimensional noetherian local domain with finite residue field, and let $E$ be a fractional subset of $D$. Then $\operatorname{Int}\left(E, D^{\prime}\right)$ is the integral closure of $\operatorname{Int}(E, D)$.

Proof. Since $\operatorname{Int}\left(E, D^{\prime}\right)$ is integrally closed, it remains to prove that it is an integral extension of $\operatorname{Int}(E, D)$ : let $f \in \operatorname{Int}\left(E, D^{\prime}\right), f(E)$ is contained in a 
$D$-algebra $R$, finitely generated as a $D$-module such that $D \subset R \subset D^{\prime}$. If $J$ is the Jacobson radical of $R$ then $J$ is the intersection of a finite number of maximal ideals with finite residue field; hence there exists an integer $q$ such that, for every $x \in R,\left(x^{q}-x\right) \in J$. If $C$ is the (nonzero) conductor $[D: R]$ and $n$ is an integer such that $J^{n} \subset C$, then for every $x$ in $R,\left(x^{q}-x\right)^{n} \in D$; hence $\left(f^{q}-f\right)^{n}=h \in \operatorname{Int}(E, D)$ and $f$ is a zero of the monic polynomial $\left(Y^{q}-Y\right)^{n}-h$ with coefficients in $\operatorname{Int}(E, D)$.

Remark 2.4. For any noetherian domain $D$ (whatever its dimension), $\operatorname{Int}\left(D, D^{\prime}\right)$ is the integral closure of $\operatorname{Int}(D)$ [19, Proposition 2.2]; once again the argument is local and can be generalized to show that $\operatorname{Int}\left(E, D^{\prime}\right)$ is the integral closure of $\operatorname{Int}(E, D)$ for many subsets $E$ of $K$ (for examples subsets of $D$ such that, for every maximal ideal $\mathfrak{m}$ of $D$ with infinite residue field, $E$ contains an infinite number of classes module $\mathfrak{m}$ ), but the question remains open in full generality. We can at least state the following.

Proposition 2.5. Let $D$ be a noetherian domain and $E$ a fractional subset of $D$. Then $\operatorname{Int}\left(E, D^{\prime}\right)$ is almost integral over $\operatorname{Int}(E, D)$.

Proof. Let $f \in \operatorname{Int}\left(E, D^{\prime}\right), f(E)$ is contained in a $D$-algebra $R$, finitely generated as a $D$-module such that $D \subset R \subset D^{\prime}$. Then, for every integer $n, f^{n}(E) \subset R$, and hence, for $d$ in the (nonzero) conductor [D:R], $d f^{n} \in \operatorname{Int}(E, D)$.

Proposition 2.3 can be improved in the case where $D$ is unibranched (generalizing a result implicitly contained in the proof of Theorem 3.1 of [19], with the same argument):

Proposition 2.6. Let $D$ be a (one-dimensional noetherian local) unibranched domain with finite residue field, and let $E$ be a fractional subset of $D$. Then $\operatorname{Int}\left(E, D^{\prime}\right)$ is a radical extension of $\operatorname{Int}(E, D):$ for any $f \in \operatorname{Int}\left(E, D^{\prime}\right)$ there is an integer $n$ such that $f^{n} \in \operatorname{Int}(E, D)$.

Proof. As above, let $f \in \operatorname{Int}\left(E, D^{\prime}\right), f(E)$ is contained in a $D$-algebra $R$, finitely generated as a $D$-module such that $D \subset R \subset D^{\prime}$. Since in this case $D$ is unibranched then $R$ is local, say with maximal ideal $\mathfrak{n}$ and some power $\mathfrak{n}^{n}$ of $\mathfrak{n}$ is contained in the (nonzero) conductor $[D: R]$, if $q$ is the cardinal of $R / \mathfrak{n}$ then, for every $x$ in $R, x^{q-1}=\varepsilon+y$ (where $\varepsilon$ is 0 or 1 and $y \in \mathfrak{n}$ ); if $p$ is the characteristic of $R / \mathfrak{n}$, it follows from a basic property of binomial coefficients that $\left(x^{q-1}\right)^{p}=\varepsilon+y$, where $\varepsilon$ is 0 or 1 and $y \in \mathfrak{n}^{2}$; by induction, letting $n=(q-1) p^{m-1}$, we have $x^{n}=\varepsilon+y$, where $y \in \mathfrak{n}^{m}$, hence $x^{n} \in D$, thus $f^{n} \in \operatorname{Int}(E, D)$.

\section{INTEGER-VALUED POLYNOMIALS OVER A UNIBRANCHED DOMAIN}

If $D$ is a local domain of maximal ideal $\mathfrak{m}$ with finite residue field and $E$ is a subset of its quotient field $K$, we let $\mathscr{I}=\{f \in \operatorname{Int}(E, D) \mid f(E) \subset \mathfrak{m}\} ; \mathscr{I}$ is clearly an ideal of $\operatorname{Int}(E, D)$. We first establish the following (generalizing [9]):

Lemma 3.1. Let $D$ be a local domain with maximal ideal $\mathfrak{m}$ and finite residue field, $E$ a subset of $K$, and $\mathfrak{M}$ a prime ideal of $\operatorname{Int}(E, D)$ containing the ideal 
$\mathscr{I}=\{f \in \operatorname{Int}(E, D) \mid f(E) \subset \mathfrak{m}\}$. Then $\mathfrak{M}$ is maximal, it is above $\mathfrak{m}$, and its residue field is isomorphic to $D / \mathrm{m}$.

Proof. $\mathfrak{M}$ is clearly above $\mathfrak{m}$, and if $q$ denotes the cardinal of $D / \mathfrak{m}$ then, for any $f$ in $\operatorname{Int}(E, D),\left(f^{q}-f\right) \in \mathscr{I}$; hence the residue field of $\mathfrak{M}$ is of cardinal $q$. Therefore, it is isomorphic to $D / \mathfrak{m}$ and $\mathfrak{M}$ is maximal.

Now if $V$ is a discrete rank-one valuation domain with finite residue field we generalize to $\operatorname{Int}(E, V)$ the classical results on the spectrum of $\operatorname{Int}(V)$ [13]. In a first step we suppose $E$ to be a subset contained in $V$ :

Lemma 3.2. Let $E$ be a subset of a rank-one discrete valuation domain $V$ with maximal ideal $\mathfrak{n}$ and with finite residue field. Then the primes of $\operatorname{Int}(E, V)$ above $\mathfrak{n}$ are in one-to-one correspondence with the elements of the topological closure $\bar{E}$ of $E$ in $\widehat{V}$ : to any element $\alpha$ of $\bar{E}$ corresponds the prime $\mathfrak{M}_{\alpha}=$ $\{f \in \operatorname{Int}(E, V) \mid f(\alpha) \in \hat{\mathfrak{n}}\}$.

Proof. This is an easy repetition of the classical proof [13]. $\mathfrak{n}$ is a principal ideal generated by an element $\pi$, hence $\mathscr{I}=\{f \in \operatorname{Int}(E, V) \mid f(E) \subset \mathfrak{n}\}=$ $\pi \operatorname{Int}(E, V)$. Now every integer-valued polynomial (on $E$ ) is a continuous function on the topological closure $\bar{E}$ of $E$ with values in the completion $\widehat{V}$ of $V$, thus

$$
\operatorname{Int}(V) \subset \operatorname{Int}(E, V) \subset \mathscr{C}(\bar{E}, \widehat{V}) .
$$

The ideal $\mathscr{I}$ is the intersection $\operatorname{Int}(E, V) \cap \mathscr{C}(\bar{E}, \hat{\mathfrak{n}})$, thus

$$
\operatorname{Int}(E, V) / \mathscr{I} \subset \mathscr{C}(\bar{E}, \widehat{V}) / \mathscr{C}(\bar{E}, \hat{\mathfrak{n}})=\mathscr{C}(\bar{E}, k)
$$

(where $\mathscr{C}(\bar{E}, k)$ is the ring of locally constant functions on $\bar{E}$ with values in $\widehat{V} / \hat{\mathfrak{n}}=k)$. Since $\bar{E}$ is compact (being a subspace of $\widehat{V}$ ), the primes of $\mathscr{C}(\bar{E}, k)$ are in one-to-one correspondence with the elements of $\bar{E}$ : to any element $x$ corresponds the set of functions null at $x$ [3, II, $\S 4$, exercise 17].

Every prime of $\operatorname{Int}(E, V)$ above $\mathfrak{n}$ contains $\pi$, hence it contains $\mathscr{I}$ and, therefore, it is maximal (Lemma 2.1); hence every prime of $\operatorname{Int}(E, V) / \mathscr{I}$ lifts in $\mathscr{C}(\bar{E}, k)$ (therefore, every prime of $\operatorname{Int}(E, V)$ lifts in $\mathscr{C}(\bar{E}, \widehat{V}))$. In conclusion the primes of $\operatorname{Int}(E, V)$ containing $\mathfrak{n}$ are of the type

$$
\mathfrak{M}_{\alpha}=\{f \in \operatorname{Int}(E, V) \mid f(\alpha) \in \hat{\mathfrak{n}}\}, \quad \text { where } \alpha \in \bar{E} .
$$

Lastly, if $\alpha \neq \beta$, there is $f$ in $\operatorname{Int}(V)$ such that $f(\alpha) \in \hat{\mathfrak{n}}$ but $f(\beta) \notin$ $\hat{\mathfrak{n}}$ (because $\operatorname{Int}(V)$ is dense in $\mathscr{C}(\widehat{V}, \widehat{V})[11,13])$, hence $\mathfrak{M}_{\alpha} \neq \mathfrak{M}_{\beta}$, since $\operatorname{Int}(V) \subset \operatorname{Int}(E, V)$.

The previous lemma generalizes to a fractional subset:

Proposition 3.3. Let $E$ be a fractional subset of a rank-one discrete valuation domain $V$ with maximal ideal $\mathfrak{n}$ and finite residue field. Then the primes of $\operatorname{Int}(E, V)$ above $\mathfrak{n}$ are in one-to-one correspondence with the elements of the topological closure $\bar{E}$ of $E$ in the completion $\widehat{K}$ of the quotient field $K$ of $V$ : to any element $\alpha$ of $\bar{E}$ corresponds the prime $\mathfrak{M}_{\alpha}=\{f \in \operatorname{Int}(E, V) \mid f(\alpha) \in \hat{\mathfrak{n}}\}$. Proof. Since $E$ is a fractional subset of $V$, there exists an element $d$ of $V$ such that $d E \subset V$; we let $F=d E$ and $\phi$ be the ring homomorphism such that $\phi(f(X))=f(X / d), \phi$ is clearly an isomorphism from $\operatorname{Int}(E, V)$ onto 
$\operatorname{Int}(F, V)$. Now $F$ is a subset of $V$ and from Lemma 3.2 the primes of $\operatorname{Int}(F, V)$ above $\mathfrak{n}$ are in one-to-one correspondence with the elements of the topological closure $\bar{F}$ of $F$ in the completion $\widehat{V}$ of $V$ (contained in the completion $\widehat{K}$ of $K$ ). If $\bar{E}$ is the topological closure of $E$ in $\widehat{K}$ then $\bar{F}=d \bar{E}$. Let $\alpha$ be an element of $\bar{F}, \beta=\alpha / d$, and $\mathfrak{N}_{\alpha}=\{f \in \operatorname{Int}(F, V) \mid f(\alpha) \in \hat{\mathfrak{n}}\}$. Then $\phi^{-1}\left(\mathfrak{N}_{\alpha}\right)=\mathfrak{M}_{\beta}=\{f \in \operatorname{Int}(E, V) \mid f(\beta) \in \hat{\mathfrak{n}}\}$.

We can generalize further to a unibranched domain:

Theorem 3.4. Let $D$ be a (one-dimensional noetherian local) unibranched domain with maximal ideal $\mathfrak{m}$ and finite residue field. Let $\mathfrak{m}^{\prime}$ be the maximal ideal of $D^{\prime}$ and $E$ be a fractional subset of $D$. The primes of $\operatorname{Int}(E, D)$ above $\mathfrak{m}$ are in one-to-one correspondence with the elements of the topological closure $\bar{E}$ of $E$ in the completion $\widehat{K}$ of $K$ for the topology defined by the valuation of $D^{\prime}:$ to any element $\alpha$ of $\bar{E}$ corresponds the prime $\mathfrak{M}_{\alpha}=\left\{f \in \operatorname{Int}(E, D) \mid f(\alpha) \in \hat{\mathfrak{m}}^{\prime}\right\}$. Proof. We consider the inclusion $\operatorname{Int}(E, D) \subset \operatorname{Int}\left(E, D^{\prime}\right)$. Since $\operatorname{Int}\left(E, D^{\prime}\right)$ is integral over $\operatorname{Int}(E, D)$ (Proposition 2.3), every prime above $m \operatorname{in} \operatorname{Int}(E, D)$ lifts in a prime above $\mathfrak{m}^{\prime}$ in $\operatorname{Int}\left(E, D^{\prime}\right)$ and hence is of the type $\mathfrak{M}_{\alpha}=\{f \in$ $\left.\operatorname{Int}(E, D) \mid f(\alpha) \in \hat{\mathfrak{m}}^{\prime}\right\}$. Moreover, if $\alpha \neq \beta$ there is $f \in \operatorname{Int}\left(E, D^{\prime}\right)$ such that $f(\alpha) \in \hat{\mathfrak{m}}^{\prime}$ but $f(\beta) \notin \hat{\mathfrak{m}}^{\prime}$ and there is an integer $n$ such that $f^{n} \in \operatorname{Int}(E, D)$ (Proposition 2.6), thus $f^{n}(\alpha) \in \hat{\mathfrak{m}}^{\prime}$ but $f^{n}(\beta) \notin \hat{\mathfrak{m}}^{\prime}$; therefore, $\mathfrak{M}_{\alpha} \neq \mathfrak{M}_{\beta}$.

Remarks 3.5. (i) If $E$ is not a fractional subset of $D^{\prime}$ then $\operatorname{Int}(E, D)=D$ since $\operatorname{Int}(E, D) \subset \operatorname{Int}\left(E, D^{\prime}\right)$ and $\operatorname{Int}\left(E, D^{\prime}\right)=D^{\prime}[19$, Lemma 2.0]. It remains to study the case where $E$ is a fractional subset of $D^{\prime}$ but not of $D$.

(ii) Theorem 3.4 does notably improve the first statement of Theorem 3.1 of [19] that says only that if $D$ is unibranched (hence $D^{\prime}$ is the ring of a valuation $v$ ) and $\alpha, \beta$ are distinct elements of $D$, then $\mathfrak{M}_{\alpha} \neq \mathfrak{M}_{\beta}$.

(iii) If $D$ is analytically irreducible, the primes of $\operatorname{Int}(D)$ above the maximal ideal $\mathfrak{m}$ of $D$ are in one-to-one correspondence with the elements of the completion $\widehat{D}$ of $D$ for the m-adic topology [11]; this special case fits with the general result of Theorem 3.4 thanks to

Proposition 3.6. Let $D$ be a (one-dimensional noetherian local) unibranched domain of maximal ideal $\mathfrak{m}$ and $\mathfrak{m}^{\prime}$ the maximal ideal of $D^{\prime}$. The following statements are equivalent:

(i) the restriction on $D$ of the $\mathrm{m}^{\prime}$-adic is the $\mathrm{m}$-adic topology;

(ii) the $\mathfrak{m}$-adic completion $\widehat{D}$ of $D$ is included in the $\mathfrak{m}^{\prime}$-adic completion $\widehat{D}^{\prime}$ of $D^{\prime}$;

(iii) $D$ is analytically irreducible.

Proof. (i) $\Rightarrow$ (ii) Clear.

(ii) $\Rightarrow$ (iii) If the completion $\widehat{D}$ of $D$ is included in the completion $\widehat{D}^{\prime}$ of $D^{\prime}$ then $\widehat{D}$ is a domain.

(iii) $\Rightarrow$ (i) If $D$ is analytically irreducible then $D^{\prime}$ is a finite $D$-module [3, 21 ], hence there is an integer $n$ such that $\mathrm{m}^{\prime n}$ is included in the (nonzero) conductor $\left[D: D^{\prime}\right]$, hence $\mathfrak{m}^{\prime n}$ is an ideal of $D$ included in $\mathfrak{m}$ and, for every $k, \mathrm{~m}^{\prime n k}$ is included in $\mathrm{m}^{k}$. On the other hand, there is an integer $m$ such that $\mathfrak{m}^{m}$ is included in $\mathfrak{m}^{\prime n}$ (since $D$ is one-dimensional noetherian and local) and, for every $k, \mathrm{~m}^{m k}$ is included in $\mathrm{m}^{\prime n k}$. 


\section{NOETHERIAN PROPERTY}

We generalize to $\operatorname{Int}(E, D)$ the results on the noetherian property of $\operatorname{Int}(D)$ [19]; as in this particular case, an obvious necessary condition for $\operatorname{Int}(E, D)$ to be noetherian is for $D$ itself to be noetherian (since $D$ is the homomorphic image of $\operatorname{Int}(E, D)$ by the evaluation map at some element $\alpha$ of $E$ ). We set first the case of a finite subset $E$.

Lemma 4.1. Let $D$ be a domain and $E$ a finite subset of $K$. Then $\operatorname{Int}(E, D)$ is not noetherian.

Proof. If $E$ contains only one point $\alpha$ then clearly $\operatorname{Int}(E, D)=D+$ $(X-\alpha) K[X]$; it shares with $K[X]$ the ideal $I=(X-\alpha) K[X]$ and it is not noetherian since $K=K[X] / I$ is not finitely generated (as a module) over $D=\operatorname{Int}(E, D) / I\left[9\right.$, Proposition 1]. If $E$ contains the points $\alpha_{1}, \ldots, \alpha_{n}$ then $\operatorname{Int}(E, D)$ shares with $K[X]$ the ideal $I=\varphi K[X]$, where $\varphi=\prod_{i=1}^{n}\left(X-\alpha_{i}\right)$, and the same proof could apply (although it is less straightforward to show that $K[X] / I$ is not finitely generated over $\operatorname{Int}(E, D) / I)$. We may also show directly that $I$ is not a finitely generated ideal of $\operatorname{Int}(E, D)$ : Assume by way of contradiction that $I$ is generated by the polynomials $f_{1}, \ldots, f_{r}$. Then any polynomial $f \in I$ is such that $f=\sum_{i=1}^{r} h_{i} f_{i}$, where $h_{i} \in \operatorname{Int}(E, D)$, for $1 \leq i \leq r$. Writing $f=\varphi g$ and $f_{i}=\varphi g_{i}$ for $1 \leq i \leq r$ and dividing by $\varphi$, we have $g=\sum_{i=1}^{r} h_{i} g_{i}$; therefore, $g\left(\alpha_{1}\right)$ is in the $D$-module generated by $g_{1}\left(\alpha_{1}\right), \ldots, g_{r}\left(\alpha_{1}\right)$. We reach a contradiction since $g$ may be any polynomial of $K[X]$.

The results of the previous lemma allow us also to conclude that for an infinite fractional subset $E$ of a unibranched domain $D$, some primes of $\operatorname{Int}(E, D)$ are not finitely generated.

Lemma 4.2. Let $D$ be a (one-dimensional noetherian local) unibranched domain with finite residue field, $E$ an infinite fractional subset of $D, \mathfrak{m}^{\prime}$ the maximal ideal of $D^{\prime}$, and $\bar{E}$ the topological closure of $E$ in the completion $\widehat{K}$ of $K$ for the topology defined by the valuation of $D^{\prime}$. Then

(i) there exists a point of accumulation in $\bar{E}$ for this topology;

(ii) if $\alpha$ is a point of accumulation of $\bar{E}$ for this topology, the prime $\mathfrak{M}_{\alpha}=$ $\left\{f \in \operatorname{Int}(E, D) \mid f(\alpha) \in \hat{\mathfrak{m}}^{\prime}\right\}$ of $\operatorname{Int}(E, D)$ is not finitely generated.

Proof. (i) Since $E$ is fractional, it is a subset of the compact space $(1 / d) \bar{D}$.

(ii) Assume by way of contradiction that $\mathfrak{M}_{\alpha}$ is generated by the polynomials $f_{1}, \ldots, f_{r}$. Then $f_{i}(\alpha) \in \mathfrak{m}^{\prime}$, for $1 \leq i \leq r$, and if $\beta$ is close enough to $\alpha$ in $\bar{E}, f_{i}(\beta) \in \mathfrak{m}^{\prime}$ for $1 \leq i \leq r$. Hence the inclusion $\mathfrak{M}_{\alpha} \subset \mathfrak{M}_{\beta}$, however, $\mathfrak{M}_{\alpha}$ is maximal and distinct from $\mathfrak{M}_{\beta}$ (Theorem 3.4).

We can now generalize to $\operatorname{Int}(E, D)$ [19, Theorem 2.3].

Theorem 4.3. Let $D$ be a noetherian domain and $E$ a fractional subset of $D$. If $\operatorname{Int}(E, D)$ is noetherian then there is no height-one prime in $D^{\prime}$ with finite residue field.

Proof. Assume by way of contradiction that a maximal ideal $\mathfrak{m}^{\prime}$ of $D^{\prime}$ is heightone with finite residue field. We let $\mathfrak{m}=\mathfrak{m}^{\prime} \cap D$. There are only finitely many primes of $D^{\prime}$ above $\mathfrak{m}$ (one of them being $\mathfrak{m}^{\prime}$ ) [21, (33.10)], and there is 
a ring $R$, finitely generated as a $D$-module, such that $D \subset R \subset D^{\prime}$, and $\mathrm{m}^{\prime}$ is the only prime of $D^{\prime}$ above $\mathfrak{n}=\mathfrak{m}^{\prime} \cap R \quad(R$ is obtained by adjoining to $D$ an element which belongs to $\mathfrak{m}^{\prime}$ but to no other prime above $\mathfrak{m}$ ). Any element of the (nonzero) conductor $[D: R]$ is clearly in the conductor $[\operatorname{Int}(E, D): \operatorname{Int}(E, R)]$, hence if $\operatorname{Int}(E, D)$ is noetherian then $\operatorname{Int}(E, R)$ is a finitely generated $\operatorname{Int}(E, D)$-module and thus a noetherian domain; by localization, $\operatorname{Int}\left(E, R_{\mathfrak{n}}\right)=(\operatorname{Int}(E, R))_{\mathfrak{n}}$ (Proposition 1.5) is also noetherian. We may suppose $E$ is infinite according to Lemma 4.1, and we reach a contradiction according to Lemma 4.2 since $R_{\mathfrak{n}}$ is clearly a (one-dimensional noetherian local) unibranched domain with finite residue field.

Remarks 4.4. (i) If $E$ is not a fractional subset of $D^{\prime}$ then $\operatorname{Int}(E, D)=D$ [20, Lemma 2.0]. As above (Remark 3.5(i)), it remains to study the case where $E$ is a fractional subset of $D^{\prime}$ but not of $D$.

(ii) If $E$ is a finite subset of $D$ then it is a fractional subset.

(iii) To finish the proof of Theorem 4.3 for a subset $E$ of $D$ (without using Lemma 4.2), we could alternatively say that the integral closure of $\operatorname{Int}\left(E, R_{\mathfrak{n}}\right)$ is $\operatorname{Int}\left(E, D_{\mathrm{m}^{\prime}}^{\prime}\right)\left(\right.$ Proposition 2.3) and that $\operatorname{dim} \operatorname{Int}\left(E, R_{\mathfrak{n}}\right)=\operatorname{dim} \operatorname{Int}\left(E, D_{\mathrm{m}^{\prime}}^{\prime}\right)=2$ (Corollary 1.4). Therefore, if $\operatorname{Int}\left(E, R_{\mathfrak{n}}\right)$ is noetherian then $\operatorname{Int}\left(E, D_{\mathrm{m}^{\prime}}^{\prime}\right)$ is a dimension 2 noetherian domain [21, (33.12)]. We would then reach again a contradiction since $\operatorname{Int}\left(E, D_{\mathrm{m}^{\prime}}^{\prime}\right)$ is also a Prüfer domain, indeed, it does contain the ring of integer-valued polynomials $\operatorname{Int}\left(D_{\mathfrak{m}^{\prime}}^{\prime}\right)$ over a discrete rank-one valuation domain and such a ring is known to be a Prüfer domain [15].

A necessary condition for $\operatorname{Int}(D)$ to be noetherian, as we shall see in Corollary 4.6 , is to be included in $D^{\prime}[X]$. We can characterize this inclusion as follows (and this is a sharper version of [19, Corollary 4.4] where condition (i) is only given as sufficient).

Theorem 4.5. Let $D$ be a noetherian domain. The following statements are equivalent:

(i) every prime of height one in $D^{\prime}$ has infinite residue field;

(ii) $\operatorname{Int}\left(D, D^{\prime}\right)=D^{\prime}[X]$;

(iii) $\operatorname{Int}(D) \subset D^{\prime}[X]$;

(iv) $\operatorname{Int}\left(D^{\prime}\right)=D^{\prime}[X]$

Proof. (i) $\Rightarrow$ (ii) Let $\mathfrak{p}^{\prime}$ be a height-one prime of $D^{\prime}$ and $\mathfrak{p}=\mathfrak{p}^{\prime} \cap D$. Then $A / \mathfrak{p}$ is infinite. Indeed, either $\mathfrak{p}$ is not maximal and there is nothing to add or it is maximal, but in this case, $\mathfrak{p}^{\prime}$ is also maximal in $D^{\prime}$ and $D^{\prime} / \mathfrak{p}^{\prime}$ is a $D / \mathfrak{p}$-module of finite type $[21,(33.10)]$. Then if $f \in \operatorname{Int}\left(D, D^{\prime}\right)$, Cramer's rule shows that $f \in D_{\mathfrak{p}^{\prime}}^{\prime}[X]$. Therefore, $f \in D^{\prime}[X]$ since $D^{\prime}$ is Krull, and thus $D^{\prime}=\bigcap\left\{D_{\mathfrak{p}^{\prime}}^{\prime}: \mathfrak{p}^{\prime}\right.$ is height-one $\}$.

(ii) $\Rightarrow$ (iii) Clear from the inclusion $\operatorname{Int}(D) \subset \operatorname{Int}\left(D, D^{\prime}\right)$ (Proposition 1.1).

(iii) $\Rightarrow$ (iv) Since $D$ is noetherian, $\operatorname{Int}\left(D, D^{\prime}\right)$ is integral over $\operatorname{Int}(D)[19$, Proposition 2.2], a fortiori its elements are integral over $D^{\prime}[X]$ that is clearly integrally closed; therefore, $\operatorname{Int}\left(D^{\prime}\right) \subset \operatorname{Int}\left(D, D^{\prime}\right) \subset D^{\prime}[X]$. The reverse inclusion is clear (Proposition 1.2).

(iv) $\Rightarrow$ (i) Assume by way of contradiction that a maximal ideal $\mathrm{m}^{\prime}$ of $D^{\prime}$ has height-one with finite residue field. Then $\operatorname{Int}\left(D^{\prime}\right)=D^{\prime}[X]$ implies, by localization, that $\operatorname{Int}\left(D_{\mathfrak{m}^{\prime}}^{\prime}\right)=D_{\mathfrak{m}^{\prime}}^{\prime}[X]$ (Proposition 1.5); however, this last equality does not hold since $D_{\mathfrak{m}^{\prime}}^{\prime}$ is a discrete rank-one valuation ring. 
Theorems 4.3 and 4.5 together lead immediately to

Corollary 4.6. If $D$ is a domain such that $\operatorname{Int}(D)$ is noetherian then $D$ is noetherian and $\operatorname{Int}(D) \subset D^{\prime}[X]$.

Conversely, if $\operatorname{Int}(D) \subset D^{\prime}[X], D$ is noetherian, and $D^{\prime}$ is a finitely generated $D$-module, then $\operatorname{Int}(D)$ is noetherian.

\section{ACKNOWLEDGMENT}

The author wishes to thank the referee for several useful comments and suggestions.

\section{REFERENCES}

1. D. F. Anderson, A. Bouvier, D. E. Dobbs, and S. Kabbaj, On Jaffard domains, Exposition. Math. 5 (1988), 145-175.

2. A. Ayache and P.-J. Cahen, Anneaux vérifiant absolument l'inégalité ou la formule de la dimension, preprint 90-11 de l'URA 225, Boll. Un. Mat. Ital. B (7) (to appear).

3. N. Bourbaki, Algèbre commutative, Hermann, Paris, 1961/1965.

4. D. Brizolis, Ideals in rings of integer-valued polynomials, J. Reine Angew. Math. 285 (1976), 28-52.

5. P.-J. Cahen, Polynômes à valeurs entières, Canad. J. Math. 24 (1982), 747-754.

6. __ Couples d'anneaux partageant un idéal, Arch. Math. (Basel) 51 (1988), 505-514.

7. __ Dimension de l'anneau des polynômes à valeurs entières, Manuscripta Math. 67 (1990), 333-343.

8. __ Polynômes à valeurs entières sur un anneau non analytiquement irréductible, J. Reine Angew. Math. 418 (1991), 131-137.

9. __ Parties pleines d'un anneau noethérien, preprint 91-5 de l'URA 225, J. Algebra (to appear).

10. P.-J. Cahen and J.-L. Chabert, Coefficients et valeurs d'un polynôme, Bull. Sci. Math. (2) 95 (1971), 295-304.

11. P.-J. Cahen, F. Grazinnif, and Y. Haouat, Intégrité du complété et théorème de Stone-Weierstrass, Ann. Sci. Clermont 21 (1982), 47-58.

12. J.-L. Chabert, Anneaux des polynômes à valeurs entières et anneaux de Fatou, Bull. Soc. Math. France 99 (1971), 273-283.

13. L__ Les idéaux premiers de l'anneau des polynômes à valeurs entières, J. Reine Angew. Math. 293/294 (1977), 275-283.

14. __ Polynômes à valeurs entières ainsi que leurs dérivées, Ann. Sci. Clermont 18 (1979), 47-64.

15. Un anneau de Prüfer, J. Algebra 107 (1987), 1-16.

16. J.-L. Chabert and G. Gerboud, Polynômes à valeurs entières et binômes de Fermat, Prépublication du Labo. Math. Marseille No. 90-9, 1990.

17. R. Gilmer, Sets that determine integer-valued polynomials, J. Number Theory 33 (1989), 95-100.

18. Multiplicative ideal theory, Dekker, New York, 1970.

19. R. Gilmer, W. Heinzer, and D. Lantz, The Noetherian property in rings of integer-valued polynomials, Trans. Amer. Math. Soc. (to appear).

20. D. McQuillan, On Prüfer domains of polynomials, J. Reine Angew. Math. 358 (1985), 162-178.

21. M. Nagata, Local rings, Interscience, New York, London, and Sidney, 1962.

22. A. Ostrowski, Über ganzwertige polynome in algebraischen Zahlkörpern, J. Reine Angew. Math. 149 (1919), 117-124. 
23. G. Polya, Über ganzwertige polynome in algebraischen Zahlkörpern, J. Reine Angew. Math. 149 (1919), 97-116.

24. G. Rauzy, Ensembles arithmétiquement denses, C. R. Acad. Sci. Paris 265 (1967), 37-38.

Service de Mathematiques-Case 322, Faculte des Sciences et Techniques de St-Jérôme, 13397 Marseille Cedex 13, France

E-mail address: CAHEN@FRMOP11(Bitnet) 